\title{
LQG CONTROL OF AN HYDRAULIC ACTUATOR WITH A FLEXIBLE MECHANICAL LOAD
}

\author{
Svante Gunnarsson \\ Department of Electrical Engineering \\ Linköping University \\ S-58183 Linköping, Sweden \\ E-mail: svante@isy.liu.se
}

\author{
Petter Krus \\ Department of Mechanical Engineering \\ Linköping University \\ S-58183 Linköping, Sweden \\ E-mail: petkr@ikp.liu.se
}

\begin{abstract}
This paper presents a control system based on linear quadratic state feedback and state estimation for an hydraulic actuator with a flexible mechanical load. The purpose of the control system is to improve the dynamic properties by reducing the oscillatory behavior of the load and to eliminate the steady state error in the load position caused by external disturbances. Using a simplified linear model a control system is developed and evaluated using simulations and experiments.
\end{abstract}

Keywords. Fluid power, position control, state feedback, Kalman filters, disturbance rejection

\section{INTRODUCTION}

Systems containing fluid power components are interesting and important applications of modern automatic control theory. An example of such an application is given in this paper, where we shall study a simplified description of the crane shown in Figure 1. This is an interesting control problem also since it represents a flexible mechanical system. The flexibility of the mechanical structure has two main consequences for the behavior of the system. First, the flexibility makes it easy to cause oscillations in the structure, and second, external disturbances will cause an error in the position due to the flexibility. The aim of the control system design is hence to obtain a closed loop system such that the crane can be operated without inducing oscillations and that the crane tip can be positioned without steady state error. Even though we shall concentrate on the crane here the discussion in the paper is relevant for excavators and other types of manipulators containing fluid power components.

\section{MATHEMATICAL MODEL}

A detailed model of the crane would be difficult to derive and too complex to be used in regulator design. We shall therefore instead use a simplified model, proposed in (T.J.Viersma, 1980) and shown in Figure 2. In using this model we assume that the flexibility is restricted to the arm of the crane.

In Figure 2 the variables $p_{i}(t), A_{i}$ and $C_{i}$ denote pressure, piston area and capacitance respectively in each chamber. Furthermore $M_{1}$ and $M_{2}$ denote
Fig. 1: Hydraulic crane

masses while $B$ and $B_{P}$ represent friction coefficients and $K$ is the spring stiffness. The variables $x_{P}(t), x_{L}(t)$ and $f(t)$ finally denote piston position, load position and external force respectively. The flow into the cylinder is controlled by a valve which however not is shown in the figure. The system will now be studied using a state space description. Details concerning the derivation of such a description is given in (Gunnarsson and Krus, 1990). A natural choice of state variables is position and velocity of piston, load and valve respectively and the pressure in each chamber, i.e.

$$
\begin{aligned}
& x_{1}(t)=x_{P}(t), \quad x_{2}(t)=\dot{x}_{P}(t), \\
& x_{3}(t)=x_{L}(t), \quad x_{4}(t)=\dot{x}_{L}(t), \\
& x_{5}(t)=p_{1}(t), \quad x_{6}(t)=p_{2}(t), \\
& x_{7}(t)=x_{V}(t), \quad x_{8}(t)=\dot{x}_{V}(t) .
\end{aligned}
$$


Fig. 2: Simplified description.

Using these state variables the system can be described by

$$
\dot{x}(t)=A x(t)+B_{1} u(t)+B_{2} f(t)
$$

where $A$ is a matrix of dimension $8 \times 8$ and $B_{1}$ and $B_{2}$ are column vectors. For the feedback control of the system we shall consider the use of two output signals. These signals are piston position

$$
y_{1}(t)=x_{P}(t),
$$

and effective load pressure

$$
\begin{aligned}
y_{2}(t) & =p_{L e}(t)=p_{1}(t)-\frac{A_{2}}{A_{1}} p_{2}(t) \\
& =x_{5}(t)-\frac{A_{2}}{A_{1}} x_{6}(t)
\end{aligned}
$$

Collecting the output signals in a vector $y(t)$ gives

$$
y(t)=C x(t)
$$

where $C$ is a $2 \times 8$ matrix. For further details of the model we refer to (Gunnarsson and Krus, 1990). It should however be emphasized that the model given by equations (2) and (5) has been obtained via several simplification steps since, for example, the original system is nonlinear. We have furthermore concentrated the flexibility to the arm of the crane.

\section{LQG CONTROL}

The obvious way to control a system described by the model above is to use feedback from estimated states. See, for example, (B.Friedland, 1986). The input is then given by

$$
u(t)=-L \hat{x}(t)+l_{0} x_{r}(t)
$$

where $L$ is a row vector and $x_{r}(t)$ denotes the reference signal. We choose the vector $L$ such that the criterion

$$
J=\int_{0}^{\infty} x^{T}(t) Q_{1} x(t)+u^{T}(t) Q_{2} u(t) d t
$$

is minimized, where the design variables $Q_{1}$ and $Q_{2}$ are used to give the step response of the closed loop system desirable properties. The state estimate $\hat{x}(t)$ is obtained from the estimator (observer)

$$
\dot{\hat{x}}(t)=A \hat{x}(t)+B_{1} u(t)+K(y(t)-C \hat{x}(t))
$$

where $K$ is a $8 \times 2$ matrix. By viewing the state estimation problem in a statistical framework we can use the Kalman filter technique as a systematic method to select a suitable gain matrix $K$.

\section{LOAD DISTURBANCES}

A desirable property of the control system is that it is able to handle load disturbances, i.e. we would like the regulator to be able to position the load properly even though a constant external force is acting on the system. We shall below discuss an approach to handle this problem. Since the load position can not be measured we have to some kind of estimate of the load position. Let us start by recalling (2) and (5) and extend the state vector by letting the disturbance $f(t)$ and the integral of the piston position error be states in the model. We therefore introduce the extended state vector $X(t)$ according to

$$
X(t)=\left(x^{T}(t) \quad f(t) \quad \int_{0}^{t}\left(x_{r}(s)-x_{P}(s)\right) d s\right)^{T}
$$

where $x_{P}(t)$ and $x_{r}(t)$ denote actual and desired piston position respectively. Assuming $f(t)$ to be piecewise constant the model can be expressed as

$$
\begin{gathered}
\dot{X}(t)=\bar{A} X(t)+\bar{B}_{1} u(t)+\bar{B}_{3} x_{r}(t) \\
y(t)=\bar{C} X(t)
\end{gathered}
$$

where

$$
\begin{gathered}
\bar{A}=\left(\begin{array}{ccc}
A & B_{2} & 0 \\
0 & 0 & 0 \\
-C_{1} & 0 & 0
\end{array}\right) \quad \bar{B}_{1}=\left(\begin{array}{c}
B_{1} \\
0 \\
0
\end{array}\right) \\
\bar{B}_{3}=\left(\begin{array}{l}
0 \\
0 \\
1
\end{array}\right) \quad \bar{C}=\left(\begin{array}{lll}
C & 0 & 0
\end{array}\right)
\end{gathered}
$$

and $C_{1}$ denotes the first row of $C$. With the control signal

$$
u(t)=-\bar{L} \hat{X}(t)+l_{0} x_{r}(t)
$$

we achieve that the steady state error in piston position caused by the external force is eliminated. When the piston position is correct we know that the error in the load position is only caused by the compression of the spring, and we then have the following static relationship between the load force and the compression

$$
K \Delta_{x}(t)=K\left(x_{P}(t)-x_{L}(t)\right)=f(t)
$$


where $K$ denotes the spring stiffness. Using the spring stiffness and the estimate of the force we obtain an estimate of the compression $\Delta_{x}(t)$ from

$$
\hat{\Delta}_{x}(t)=\frac{1}{K} \hat{f}(t)
$$

This quantity can then be added to the piston position reference signal, which means that the integrator state is formed according to

$$
\dot{\hat{x}}_{n+2}(t)=\int_{0}^{t}\left(\frac{1}{K} \hat{x}_{n+1}(s)+x_{r}(s)-x_{P}(s)\right) d s
$$

With this modification we achieve a closed loop system that is able to control the load position without steady state error.

\section{REGULATOR TRANSFER FUNCTIONS}

To get a more intuitive insight into the properties of the regulator based on state feedback and state estimation it is useful to describe the feedback using transfer functions. Taking the Laplace transform of the state estimator corresponding to (10) and equation (14) yields that the feedback can be expressed as

$$
U(s)=F_{X_{r}}(s) X_{r}(s)-F_{Y}(s) Y(s)
$$

where

$$
\begin{aligned}
F_{X_{r}}(s)= & \left(l_{0}-\bar{L}\left(s I-\bar{A}+\bar{K} \bar{C}+\bar{B}_{1} \bar{L}\right)^{-1}\right. \\
& \left.\times\left(\bar{B}_{1} l_{0}+\bar{B}_{3}\right)\right)
\end{aligned}
$$

and denotes the transfer function from reference to control signal. Furthermore

$$
F_{Y}(s)=\bar{L}\left(s I-\bar{A}+\bar{K} \bar{C}+\bar{B}_{1} \bar{L}\right)^{-1} \bar{K}
$$

and since $K$ is a matrix with two columns $F_{Y}(s)$ becomes a row vector with two transfer functions as elements. We can hence rewrite (18) as

$U(s)=F_{X_{r}}(s) X_{r}(s)-F_{X_{P}}(s) X_{p}(s)-F_{P_{L e}}(s) P_{L e}(s)$

where $F_{X_{p}}(s)$ and $F_{P_{L e}}(s)$ denote the transfer functions from piston position and effective load pressure respectively to control signal.

Our next step is then to apply the LQG design procedure presented above to the crane model with the model parameters chosen such that the model gives a reasonable description of the true system and with design variables giving the closed loop system desirable properties. For details we refer to (Gunnarsson and Krus, 1991). By then plotting the Bode diagrams of the transfer functions in the feedback given by equation (19) we find that the transfer functions have surprisingly simple character despite the rather high model order.
Before we discuss these transfer functions further we will however rewrite equation (21) slightly. It is desirable to have the integral action just before the computation of the control signal in order to be able to incorporate an anti-windup mechanism. By therefore moving $F_{X_{r}}(s)$ into the loop and introducing

$$
\begin{aligned}
\bar{F}_{X_{p}}(s) & =F_{X_{p}}(s) / F_{X_{r}}(s) \\
\bar{F}_{P_{L e}}(s) & =F_{P_{L e}}(s) / F_{X_{r}}(s)
\end{aligned}
$$

we can express feedback as

$$
\begin{aligned}
U(s) & =F_{X_{r}}(s)\left[X_{r}(s)\right. \\
& \left.-\bar{F}_{X_{p}}(s) X_{p}(s)-\bar{F}_{P_{L e}}(s) P_{L e}(s)\right]
\end{aligned}
$$

Evaluating the transfer functions in (23) the chosen model and design parameters shows that $F_{X_{r}}(s)$ has the properties of a PI-regulator. The feedback from piston position $\bar{F}_{X_{p}}(s)$ is also of simple character and can be seen as a first order lead compensator is series with a first order low pass filter. The total piston position feedback hence becomes a PID-regulator in series with a second order low pass filter. Finally the pressure feedback $F_{P_{L e}}(s)$ is a band pass filter having zero static gain and with the pass band located at the resonance frequency of the system.

\section{EXPERIMENTS}

Since it would be difficult to obtain good estimates of all parameters in the crane model we will utilize that the regulator transfer functions can be approximated by transfer functions of lower order with properties related to, for example, the resonance frequency of the structure. In the real system the transfer function varies with operating point and load, but we will here for simplicity study the system in the vicinity of one operating point. The low order approximate LQG-regulator has been implemented on the computer controlling the real crane, and the performance of the control system has been tested by making a step change in the reference position. The load position is of course of primary interest but since this signal cannot be measured we concentrate on the piston position. Some information concerning the dynamic behavior of the load can be obtain via the pressure signal, since the oscillations in the load cause oscillations in the oil pressure. We will here concentrate on two experiments, and these experiments have two purposes. First we wish to validate our linear model by showing that the simulation model and the real system gives comparable results under similar conditions. Second we wish to show that the (approximate) LQG regulator results in a closed loop system with good properties. In the first experiment we use only proportional feedback of the piston position. The result from the linear 
model and the real crane are shown in Figures 3 and 4 . Figures 5 and 6 then shows the step responses when the LQG feedback is used. From these figures we can draw the conclusions that the LQG feedback gives a faster closed loop system and improved damping. The improved damping is seen also in the pressure signal. We also see that the results from the linear model coincide well with the experimental results. The response of the linear model is somewhat faster but the agreement is satisfactory with respect to the high degree of simplification in the linear model.

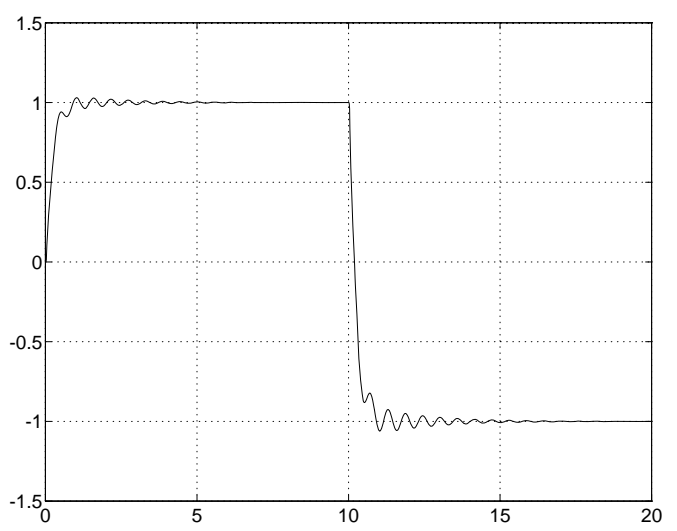

Fig. 3: Piston position step response in simulation model

Fig. 4: Solid - Piston position step response in real system. Dotted - Pressure signal

\section{CONCLUSIONS}

We have shown how an approximate LQG regulator, designed using a linear model, can be used to control an hydraulic actuator with a flexible mechanical load. The control system is designed such that the closed loop system gets a well damped behavior and such that the piston position is controlled without steady state error in case of external disturbances. We have also indicated a method

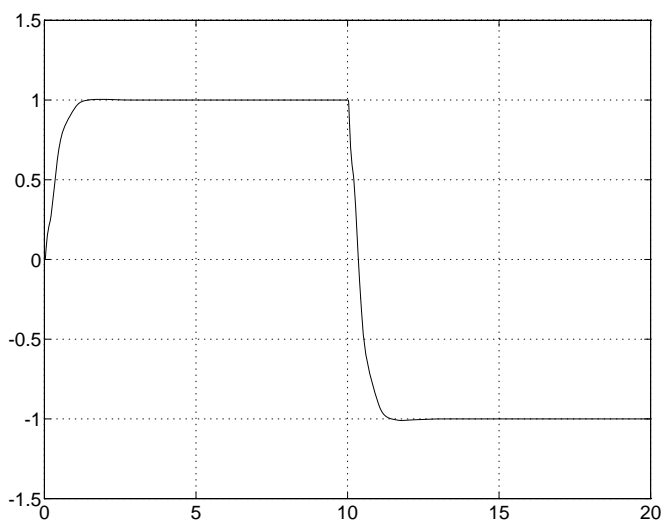

Fig. 5: Piston position step response in simulation model

Fig. 6: Solid - Piston position step response in real system. Dotted - Pressure signal

for compensating the error in the load position caused by external disturbances.

\section{REFERENCES}

B.Friedland (1986). Control system design. An introduction to state space methods. McGrawHill, New York.

Gunnarsson, S. and Krus, P. (1990). "Modelling of a flexible mechanical system containing hydraulic actuators". Technical report, LiTHISY-1078, Department of Electrical Engineering, Linköping University, Linköping, Sweden.

Gunnarsson, S. and Krus, P. (1991). "LQG control of an hydraulic actuator with a flexible mechanical load". Technical report, LiTH-ISY1209, Department of Electrical Engineering, Linköping University, Linköping, Sweden.

T.J.Viersma (1980). Analysis, synthesis and design of hydraulic servosystems and pipelines. Elsevier Scientific Publishing Company, Amsterdam, The Netherlands. 